\title{
ESTABLISHMENT OF AIR PISTON GAUGE AS PRIMARY PRESSURE STANDARD AT CSIR-NATIONAL PHYSICAL LABORATORY INDIA
}

\author{
Vikas N. Thakur ${ }^{1,2 a}$, Sanjay Yadav ${ }^{1,2 b}$, Ashok Kumar ${ }^{1,2 c}$ \\ ${ }^{1}$ CSIR-National Physical Laboratory, Dr. K. S. Krishnan Marg, Delhi 110012, India \\ ${ }^{2}$ Academy of Scientific and Innovative Research (AcSIR), Ghaziabad-201002, India \\ a vikasnthakur92@gmail.com, ${ }^{\mathrm{b}}$ syadav@nplindia.org, ${ }^{\mathrm{c}}$ ashok553@nplindia.org
}

\section{Abstract:}

The air piston gauge (APG) was established at CSIR-National Physical Laboratory, India (NPLI) since 2000. Later the same piston-cylinder(p-c) assembly was calibrated in NIST USA; however, it was never published for metrology communities. As per international protocol, the establishment of the APG as a primary standard, the effective area of pc assembly, and masses must be directly traceable to SI units. The first time we have calculated the effective area and associated uncertainty of $p-c$ assembly using dimension and mass metrology, traceability to the SI units, i.e., meter and kilogram. To realize the APG as primary pressure standards, we have calculated the effective area of $p$-c assembly of APG directly from dimension metrology, which is further supported by various other methods. The effective area values obtained in the pressure range of $6.5-360 \mathrm{kPa}$ lie in the range of $3.356729-3.357248 \mathrm{~cm}^{2}$ due to uncertainty limitation in the measurement of dimension of internal diameter of cylinder. The expected values of the effective area which are also measured from cross-float technique against ultrasonic interferometer manometer (UIM), primary pressure standards. The accuracy in effective area measurement is possible only when the resolution in the internal radius of the cylinder should at least be up to 5 th decimal order and the uncertainty is $80 \mathrm{~nm}$. The expanded uncertainty was measured nearly 11 ppm at $k=2$ by considering the uncertainty in internal radii of cylinder and radii of piston around $80 \mathrm{~nm}$.

Keywords: Uncertainty; air piston gauge; pressure; primary standard

\section{INTRODUCTION}

The most accurate pressure standard all over world is mercury based manometer i.e. ultrasonic interferometer manometer (UIM). Due to toxic nature of mercury and complications in handling the electronics and working of the whole UIM most of the national metrology institutes (NMIs) have decommissioned the UIM. Recently national institute of standards and technology (NIST), USA also decommissioned the UIM; however, NPLI still maintained the same and recently participated in APMP.M.P-K4 key comparisons. Since most of the NMIs have banned mercury based products including UIM, now focus has shifted to APG with precision and high accuracy measurement in the radii/effective area of p-c assembly. Various NMIs have been developing a piston-cylinder (p-c) assembly as a primary pressure standard to replace the ultrasonic interferometer manometer. The p-c assembly can be converted into primary pressure standards using calibrated mass and dimensions form mass and dimension metrology, respectively. The NPLI has functional primary pressure standards, UIM, which has the relative expanded uncertainty of $7.2 \mathrm{ppm}$ at $\mathrm{k}=2$ in the pressure range of $1 \mathrm{~Pa}-$ $130 \mathrm{kPa}$ established in 1982 and maintained since then [1].

Recently, NIST USA has been developed laser interferometer based Optical Interferometer Manometer (OIM) primary pressure standards [24]. To meet the requirement of mercury-free equipment, most of the NMIs has developed p-c assembly based primary pressure standards to realize the Pascal and maintain the traceability chain. The APGs are easy to handle, portable in nature, and very accurate pressure measurement over a wide range of pneumatic and hydraulic pressure, i.e., $\mathrm{Pa}$ to GPa. In this article, our focus will be on the calculation of the effective area of p-c assembly using rarefaction gas dynamics and dimension metrology.

To calculate the effective area, the theory of rarefaction gas dynamics has been used. A formula was derived for calculating the effective area, which is a combined form of viscous and free-molecular flow of gas in a medium [5-8]. The NIST USA, and PTB Germany have discarded the Dadson formula to calculate the effective area of p-c assembly and opted to use the rarefaction gas dynamics method. 
Here we report for the values of the effective area along with the uncertainties of the APG, model: Ruska 2465A-754 using dimension metrology and rarefaction gas dynamics theory in the range of 6.5 $-360 \mathrm{kPa}$.

\section{EFFECTIVE AREA CALCULATION}

In this method, we have taken the dimensional measurements of $p-c$ assembly like diameters of piston and cylinder at various angles in the range of $0^{\circ}-360^{\circ}$ along with the roundness and straightness at different heights. Since the roundness and straightness are very low and within their uncertainty limits. Therefore, the p-c assembly was considered to be ideally straight and circular. Figure 1 illustrates the schematic diagram of the $p$ - $c$ assembly for calculating the effective area using the dimension of $p-c$ assembly. Figure 1 explains the cross-section of the $p-c$ cylinder assembly.
Horizontal dash lines: PQ and GH in Figure 1 are the extreme ends of engagement length ' $l$ ' which are indicated by $x=0$ and $x=l . x$ is a vertical axis coordinate in the range of $[0, l]$. Vertical dash lines: $\mathrm{AB}$ is the ideal line for cylinder, and $\mathrm{CD}$ and $\mathrm{EF}$ are the ideal lines for the piston. The distance between dashed lines TU and EF is ideal radius of the piston $\left(r_{o}\right)$. The ideal crevice size is given by $h_{o}$. The ideal radius of the internal surface of the cylinder is given by $R_{o}=r_{o}+h_{o}$. Solid curves: RS and TU are the imaginary curves (shapes in 3D) of the internal surface of the cylinder (on the left side) and piston (on the right side), respectively. The effective area at given temperature $\left(A_{e}\right)$ was calculated. Since in theoretical calculation i.e. RGD, zero pressure effective area and $A_{e}$ are having negligible or no difference.

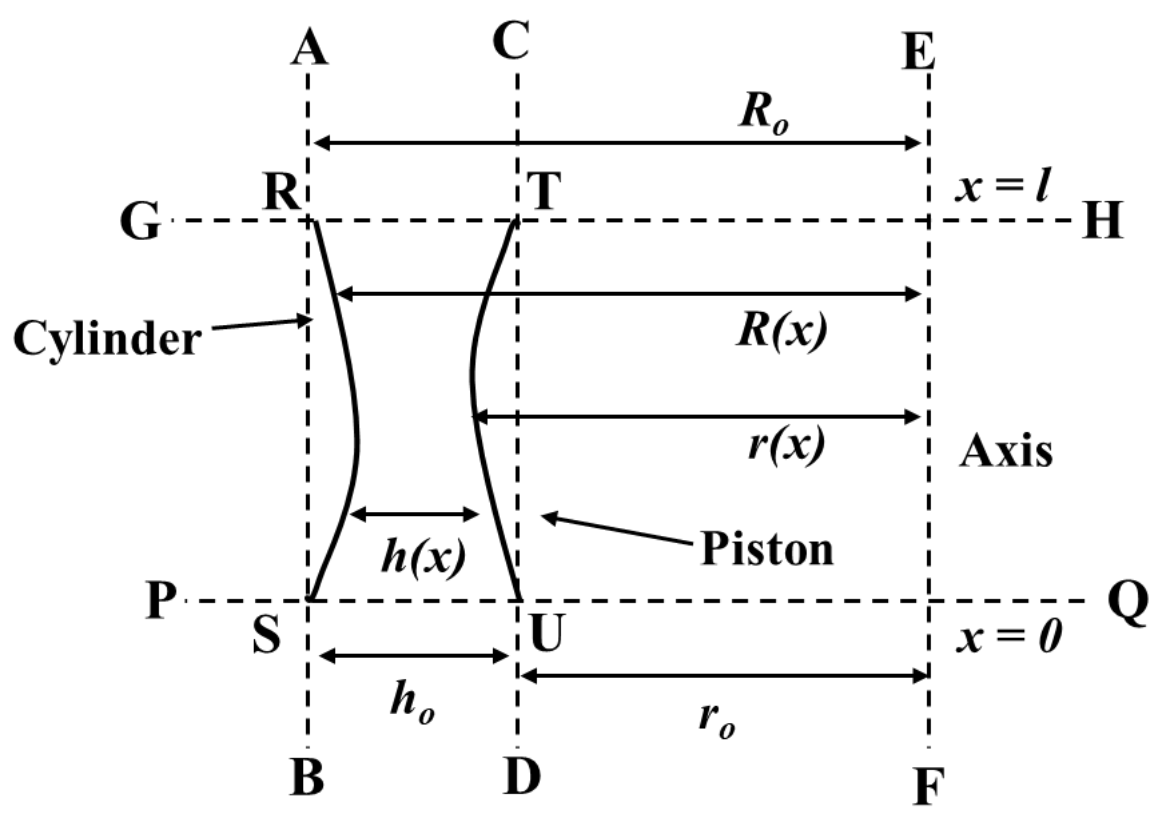

Figure 1: 2D cross-section image was drawn for the detailed explanation about the physical parameters.

The formula to calculate effective area $\left(A_{0}\right)$ of $p$ - $c$ assembly given by equation (1) has three contributions: (1) $A_{l}$ is due to the force acting on the piston due to the net pressure, i.e. $\left(\triangle P=P_{1}-P_{2}\right)$, where $P_{1}$ is the applied pressure at the bottom of the piston and $P_{2}$ is the ambient pressure (given by equation (1a)), (2) $A_{2}$ is due to the drag force due to the pressure flow in the gap between the piston and cylinder (given by equation (1b))and (3) $A_{3}$ represents the area affected due to the variation of the piston radius (given by equation (1c)).

$$
\begin{aligned}
& A_{0}=A_{1}+A_{2}+A_{3} \\
& A_{1}=\frac{\pi\left(r^{2}(x=0) P_{1}-r^{2}(x=l) P_{2}\right)}{P_{1}-P_{2}}
\end{aligned}
$$

$$
\begin{aligned}
& A_{2}=-\frac{\pi}{\left(P_{1}-P_{2}\right)} \int_{0}^{l} r(x) h(x) \frac{d P}{d x} d x \\
& A_{3}=\frac{2 \pi}{\left(P_{1}-P_{2}\right)} \int_{0}^{l} r(x) P(x) \frac{d r(x)}{d x} d x,
\end{aligned}
$$

where, $P(x)$ is the pressure distribution along the clearance/gap between the piston and cylinder, $h(x)=r(x)-R(x)$ is the radial clearances between piston and cylinder at $x=0$ and along with the radial axis, respectively, $r_{o}$ and $R_{o}$ are the ideal radii of the piston and cylinder at $x=0$ and $r$ and $R$ are radii of piston and cylinder, respectively with varying engagement length $(x)$. To calculate $A_{2}$ and $A_{3}$, pressure distribution has to be calculated 
using the theories developed in the references ${ }^{3,4}$. The gas flow rate in the gap between the piston and cylinder is given by equation (2),

$$
\text { Gas flow rate }=-\frac{2 \pi r_{o}}{v_{m p s}} G_{P}(\delta) h^{2}(x) \frac{d P}{d x}
$$

where $G_{P}(\delta)$ is Poiseuille's coefficient which is function of rarefaction parameter $(\delta=P(x) h(x) /$ $\left.\left(\eta v_{m p s}\right)\right)^{3,4}$, where $\eta$ is the coefficient of viscosity of gas i.e. $17.72 \mu \mathrm{Pa}$ sec for $\mathrm{N}_{2}$ [9] and $v_{m p s}=$ $\sqrt{2 R_{g} T / m}$ is the most probable speed, $R_{g}$ is the universal gas constant, $m$ is the molecular mass of the gas used and $T$ is the temperature inside APG.

$\delta(x)$ is dependent on the pressure in the crevice $P(x)$. The pressure distribution is calculated using iteration method by varying crevice size $(h(x))$. After calculating pressure distribution and crevice size, rarefaction parameter was calculated. The rarefaction parameter was further used to calculate Poisuelle's coefficient $\left(G_{P}(\delta)\right.$.by considering crevice as planar channel using kinetic Boltzmann equation $^{3,4}$ In case of the diffuse scattering of molecules on the crevice wall, the Poiseuille coefficient is given as

$$
\begin{gathered}
G_{p}(\delta)=3.869+\frac{1-\alpha_{t}}{\alpha_{t}} \text { for } \delta \\
<2.13 \times 10^{-3} \\
G_{p}(\delta)=\frac{-\ln \delta}{\sqrt{\pi}}+0.376-(1.77 \ln \delta+0.584) \delta \\
+2.12 \delta^{2}+\frac{1-\alpha_{t}}{\alpha_{t}} \\
\text { for } 2.13 \times 10^{-3}<\delta<0.45 \\
G_{p}(\delta)=\frac{\delta}{6}+\sigma_{p}+\frac{0.61}{\delta^{0.7}}-\frac{0.24}{\delta} \\
+\frac{1-\alpha_{t}}{\alpha_{t}} \text { for } \delta \\
>0.45
\end{gathered}
$$

where $\sigma_{p}=1.018$ is the slip coefficient [11]. In real sense, $\mathrm{N}_{2}$ gas does not have the complete accommodation, but its tangential component of accommodation coefficients is given by $\alpha_{t}=0.9$ [10]. Thus, $G_{p}(\delta)$ should be corrected as

$G_{p}\left(\alpha_{t}\right)=G_{p}(1)+1.772 \frac{1-\alpha_{t}}{\alpha_{t}}$

After getting Poiseuille's coefficient and crevice size we get the pressure distribution in crevice given by equation (4)

$P(x)=P_{1}-\left(P_{1}-P_{2}\right) \frac{\int_{0}^{x}\left(h(x)^{2} G_{P}(\delta)\right)^{-1} d x}{\int_{0}^{l}\left(h(x)^{2} G_{P}(\delta)\right)^{-1} d x}$
This pressure distribution is used to calculate the effective area using equation (1) to (1c) with varying applied pressure in the range of $6.5 \mathrm{kPa}$ to $360 \mathrm{kPa}$ with the associated uncertainty.

\section{RESULTS \& DISCUSSION}

The radii of the piston and cylinder are given in Table 1 along with their associated uncertainty. Based on dimension metrology data, RGD theory was used to calculate the effective area values of $p$ c assembly pressure range of APG and obtained $3.356729-3.357248 \mathrm{~cm}^{2}$ with varying internal radius of cylinder within the uncertainty limit given by dimensional metrology i.e. $700 \mathrm{~nm}$ (shown in Figure 2). The effective area found in cross float method considered to be the expected values of effective area. While varying the value of the internal diameter of the cylinder, the expected value of effective area i.e. $3.356770-3.356780 \mathrm{~cm}^{2}$ was found which is at internal diameter $=10.3380 \mathrm{~mm}$ (shown in Figure 2(b)). This value of internal radius of cylinder is $650 \mathrm{~nm}$ away from the value given by the dimensional metrology given in Table 1.

Table 1: Radii of the piston and internal surface of the cylinder@ $23{ }^{\circ} \mathrm{C}$.

\begin{tabular}{|l|l|l|}
\hline Part of APG & $\begin{array}{l}\text { Radius } \\
(\mathrm{mm})\end{array}$ & $\begin{array}{l}\text { Uncertainty } \\
(\mathrm{nm})\end{array}$ \\
\hline Piston & 10.33561 & 80 \\
\hline $\begin{array}{l}\text { Internal surface of } \\
\text { the cylinder }\end{array}$ & 10.3386 & 700 \\
\hline
\end{tabular}

The expected values of effective area was found at internal diameter of cylinder $(R)=10.3380 \mathrm{~mm}$ within uncertainty limits of $80 \mathrm{~nm}$. The values of effective area using $R=10.3380 \mathrm{~mm}$ is shown in Figure 2(b) which are in the range of the effective area values obtained since last 20 years from crossfloat against ultrasonic interferometer manometer (UIM) [11]. Figure 2(b) also shows that the effective area values can be achieved with high accuracy only when atleast the radius of the internal surface of the cylinder has the resolution upto $5^{\text {th }}$ decimal place an uncertainty should be within 80 $\mathrm{nm}$. Then the zero pressure effective area was also calculated using RGD which is the value at constant temperature and pressure. The zero pressure effective area values obtained from RGD and crossfloat against UIM are $3.356775 \mathrm{~cm}^{2}$ and 3.356772 $\mathrm{cm}^{2}$, respectively. The standard deviation between these two values of zero pressure effective area is $2 \times 10^{-6} \mathrm{~cm}^{2}$. The uncertainty in the measurement of zero pressure effective area was calculated using equation (4) by considering $80 \mathrm{~nm}$ uncertainty in both piston and cylinder radii. 

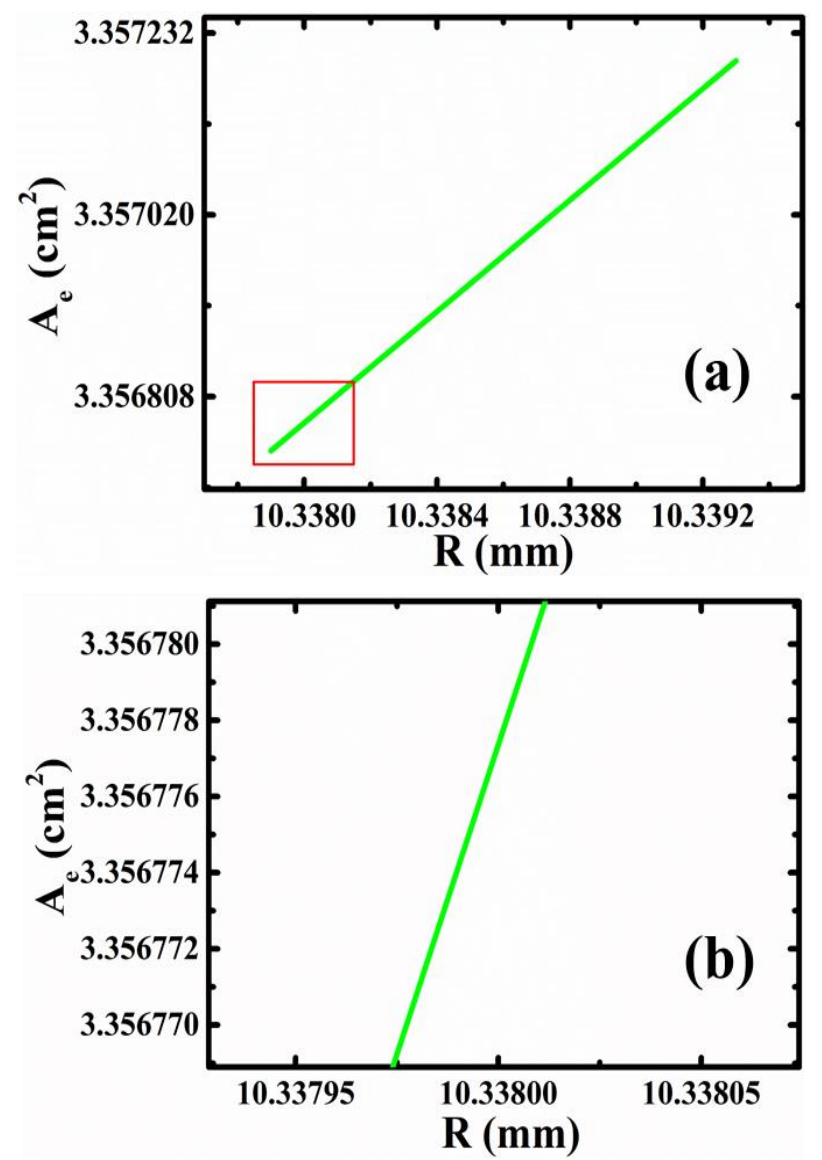

Figure 2: (a) Effective area values with varying radius of internal surface of cylinder with in its uncertainty limits, (b) The region bounded in Figure 2(a) is shown as magnified image.

The expanded relative uncertainty in the zero pressure effective area was calculated to be $11 \mathrm{ppm}$ of the reading at $k=2$.

$\frac{u\left(A_{0}\right)}{A_{0}}=\frac{\sqrt{u\left(r_{0}\right)^{2}+u\left(R_{0}\right)^{2}}}{2 r_{\text {avo }}}$

where $r_{a v o}$ is the average radius of the piston and internal surface cylinder radii.

\section{SUMMARY}

The effective area values of APG obtained using RGD is in the range of $3.356729-3.357248 \mathrm{~cm}^{2}$ with varying internal cylinder radius within its uncertainty limit i.e. $700 \mathrm{~nm}$ in the complete pressure range of $6.5-360 \mathrm{kPa}$. But the expected values of the effective area i.e. $3.356770-3.356780$ $\mathrm{cm}^{2}$ were obtained at $10.33797 \mathrm{~mm}$ to $10.33801 \mathrm{~mm}$ internal cylinder radius within the uncertainty limit less than $80 \mathrm{~nm}$. The accuracy and low uncertainty is possible only when the resolution in the internal radius of the cylinder should have atleast be upto $5^{\text {th }}$ decimal and the uncertainty is comparable to uncertainty of pistion i.e. $80 \mathrm{~nm}$ in the present case. The expanded relative uncertainty in zero pressure effective area was also calculated by considering 80 $\mathrm{nm}$ as the uncertainty in both internal cylinder and piston radii. The expanded uncertainty of $11 \mathrm{ppm}$ at $k=2$ was calculated in the zero pressure effective area. The value of expanded uncertainty obtained from RGD in zero pressure effective area is comparable to that of the cross-floating against UIM. The zero pressure effective area values obtained from cross float against UIM and RGD have standard deviation of $2 \times \mathbf{1 0}^{-6} \mathrm{~cm}^{2}$.

\section{ACKNOWLEDGEMENTS}

Authors would like to thank Dr. Felix Sharipov and Dr. Y. Yang, for scientific guidance to undersatnd the RGD model.

\section{REFERENCES}

[1] A. Kumar; V. N. Thakur; R. Sharma; H. Kumar; Omprakash; S. Yadav, Uncertainty evaluation and phase variation of ultrasonic interferometer manometer: A primary pressure and vacuum standard, Vacuum. 165 (2019) 232-238. https://doi.org/10.1016/j.vacuum.2019.04.023.

[2] K. Jousten, J. Hendricks, D. Barker, K. Douglas, S. Eckel, P. Egan, J. Fedchak, J. Flügge, C. Gaiser, D. Olson, J. Ricker, T. Rubin, W. Sabuga, J. Scherschligt, R. Schödel, U. Sterr, J. Stone, G. Strouse, Perspectives for a new realization of the pascal by optical methods, Metrologia. 54 (2017) S146-S161.

https://doi.org/10.1088/1681-7575/aa8a4d.

[3] Y. Takei, K. Arai, H. Yoshida, Y. Bitou, S. Telada, T. Kobata, Development of an optical pressure measurement system using an external cavity diode laser with a wide tunable frequency range, Measurement. 151 (2020) 107090.

https://doi.org/10.1016/j.measurement.2019.10709 0 .

[4] P.F. Egan, J.A. Stone, J.H. Hendricks, J.E. Ricker, G.E. Scace, G.F. Strouse, Performance of a dual Fabry-Perot cavity refractometer, Opt. Lett. 40 (2015) 3945.

https://doi.org/10.1364/OL.40.003945.

[5] Felix Sharipov, Rarefied Gas Dynamics: Fundamentals for Research and Practice, Wiley, Berlin, 2016.

[6] F. Sharipov, Y. Yang, J.E. Ricker, J.H. Hendricks, Primary pressure standard based on piston-cylinder assemblies. Calculation of effective cross sectional area based on rarefied gas dynamics, Metrologia. 53 (2016) 1177-1184.

https://doi.org/10.1088/0026-1394/53/5/1177.

[7] F. Sharipov, V. Seleznev, Data on internal rarefied gas flows, J. Phys. Chem. Ref. Data. 27 (1998) 657-706. https://doi.org/10.1063/1.556019.

[8] W. Sabuga; F. Sharipov; and T. Priruenrom, Determination of the effective area of pistoncylinder assemblies using rarefied gas flow model, in: PTB-Mitteilungen 5th CCM Int. Conf. Press. Vac. Metrol., 2011: pp. 260-2.

[9] W.A. Cole, W.A. Wakeham, The Viscosity of Nitrogen, Oxygen, and Their Binary Mixtures in 
the Limit of Zero Density, J. Phys. Chem. Ref. Data. 14 (1985) 209-226.

https://doi.org/10.1063/1.555748.

[10] F. Sharipov, Application of the Cercignani-Lampis scattering kernel to calculations of rarefield gas flows. I. Plane flow between two parallel plates, Eur. J. Mech. B/Fluids. 21 (2002) 113-123. https://doi.org/10.1016/S0997-7546(01)01160-8.
[11] Vikas N. Thakur; R. Sharma; H.Kumar; Omprakash; D. A. Vijaykumar; S. Yadav; A. Kumar, On long-term stability of an air piston gauge maintained at National Physical Laboratory, India, Vacuum. 176 (2020) 109357. https://doi.org/10.1016/j.vacuum.2020.109357. 